\title{
A Methodology for Modeling a Quality Embedded Remanufacturing System
}

\author{
Youngseok Kim, Hong-Bae Jun, Dimitris Kiritsis, and Paul Xirouchakis \\ EPFL, STI-IPR-LICP, ME B1, Station 9, 1015 Lausanne, Switzerland, \\ youngseok.kim@epfl.ch \\ WWW home page: http://licpwww.epfl.ch
}

\begin{abstract}
The uncertain quality of used products highly affects the performances of remanufacturing systems and quality of remanufactured products. Hence the quality issues of used product cannot be neglected in remanufacturing. To apply the quality concept into remanufacturing system control or simulation, individual management of each product is required. To this end, a multi-agent approach can provide good solutions. The first step in applying the quality concept with the multi-agent approach is an effective modeling of a remanufacturing system. This study proposes a methodology for modeling a quality embedded remanufacturing system (QRS) with two layers. The first layer represents elements in a remanufacturing system as it is. The representation also contains the information of a multi-agent structure for a QRS. The second layer expresses the status of each product and resource agent, and their relationships to manage the multi-agent system. As modeling tools to support the proposed methodology, directed graphs and Petri-Nets are used. A case study is introduced to show an application of the proposed methodology.
\end{abstract}

\section{Keywords}

Remanufacturing, Modeling methodology, Agent, Petri-Nets, Quality

\section{Introduction}

Used products are basic elements in remanufacturing. The uncertain quality of used products and components/parts which are disassembled from the used products highly affect the performances of remanufacturing systems and the qualities of remanufactured products [1]. Hence quality issues cannot be neglected in remanufacturing. To reflect the uncertain quality status of parts (for simplicity, in this study 'part' is a general term for a used product, a disassembled component/part, 
and a reassembled product) to the remanufacturing system control or simulation, each part has to be managed individually. Until now, it has not been easy to manage each part individually considering its characteristics, because there was no way to identify each part in a remanufacturing system. But emerging product identification technologies like radio frequency identification (RFID) make it possible.

This study focuses on the modeling methodology for a quality embedded remanufacturing system (QRS). The modeling is important because all analysis, management, control, and simulation works are carried out based on the modeled information. To manage parts and resources individually in a remanufacturing line, this study applies a multi-agent framework where each part and resource are regarded as an agent, respectively. The agent approach has been recognized as an effective solution to overcome the traditional approaches' limits in abilities of the expansion, reconfiguration, maintenance without shut down, and so on [2]. Defining each part as an agent can also enrich the individual controllability of each part, because remanufacturing systems usually handle multiple kinds of products.

The remaining of this study is as follows. Section 2 reviews previous related research. Section 3 represents necessary modeling elements in a remanufacturing system. Section 4 proposes a modeling methodology and proper modeling tools. Finally, section 5 introduces a case study to show how the proposed methodology can be applied to a real case.

\section{Previous Related Research}

Most previous research on remanufacturing systems focus on the disassembly phase which highly involves quality uncertainty and parts composition diversity of used products. They usually borrow ideas of graph representation from research on assembly modeling tools. Demello and Sanderson [3] analyzed optimal assembly/disassembly sequences with AND/OR graphs, in which all possible components and parts are marked as nodes and assembly/disassembly relationships as arcs. Zussman and Zhou [4] proposed disassembly Petri-Nets (DPN). They formally imported the concept of disassembly operation quality into their tool by inserting quality test transitions right after each transition for a disassembly action.

Modeling tools for simulation or control of manufacturing/remanufacturing systems have been considered. In the simulation or control, their logic and information are usually managed based on system status; a simulator collects and analyzes information of status change of manufacturing/remanufacturing systems, and a controller decides the next status of the system depending on its current status. Such systems can be regarded as a kind of an automaton system. Petri-Nets are widely used for representing these automata systems because of their well known efficiency in managing system status. For example, EIMekkawy and ElMaraghy [5] used timed Petri-Nets (TPN) to develop a rescheduling algorithm. Jeng et al. [6] handled a resource sharing with process nets with resources (PNRs). Furthermore, as a more generalized simulation tool, CIMPACT developed software, SIMAS [7], which enables a simulation of manufacturing/remanufacturing systems from the perspective of processing time. 
Among previous research on modeling, extended two-level colored Petri-Nets (XCPN) [8] can be considered as a suitable modeling tool for a remanufacturing system modeling because of the following reasons. First, the modularization concept of the tool enables separated modeling of resources and products. Second, the color, inherited from colored Petri-Nets (CPN), and time characteristics make stochastic simulation possible, which is necessary in simulating the stochastic characteristics of remanufacturing systems. However, a small modification is required because the $\mathrm{XCPN}$ lacks the characteristics for handling the quality dependent part routing.

As explored above, although there are many research works on modeling tools, there is lack of modeling tools for remanufacturing system simulation or control incorporating the quality concept. To our knowledge, there is no literature that explains an overall methodology of modeling remanufacturing systems. To overcome these limitations, this study proposes a structured modeling methodology and an overall outline of suitable modeling tools for the methodology.

\section{Modeling Elements}

To represent a QRS well, necessary elements have to be extracted. To this end, we apply a top-down approach. In this approach, we inspect necessary elements composing the QRS, and their sub elements until the bottom level.

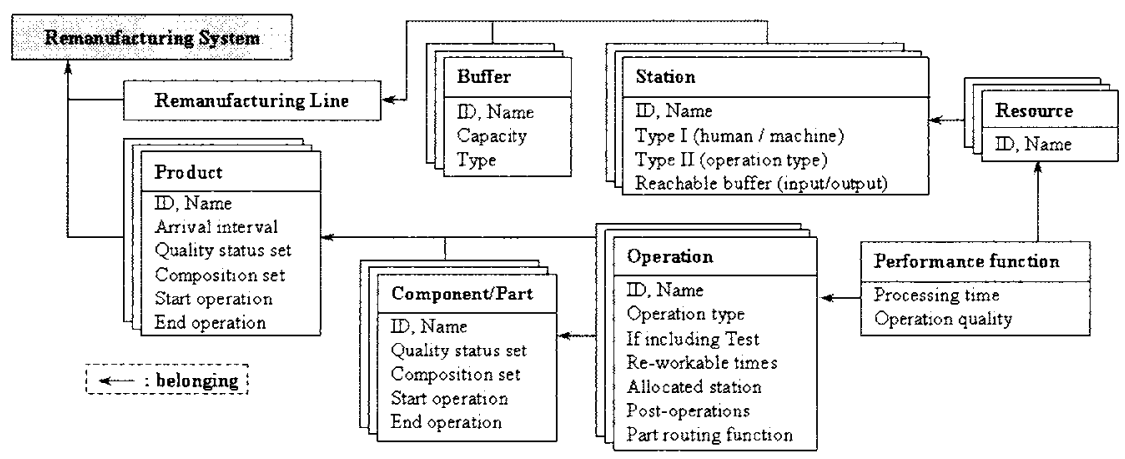

Fig. 1. Elements in a quality embedded remanufacturing system (QRS).

Fig. 1 shows the extracted elements and their attributes. Two main elements in a remanufacturing system are a remanufacturing line and products which flow on the line. The line comprises buffers and work stations which have some resources. A remanufacturing system can handle multiple kinds of products which are composed of some parts. Each product and disassembled part is assigned with operations to be processed.

To simulate or control a remanufacturing system, performance related information of each operation is required, e.g. processing time and operation quality. Such performance indices are affected not only by an operation but also by a 
resource which is in charge of the operation, because even the same operation can show different performance depending on a resource's type, degree of expertise, degree of fatigue, and so on. Hence the performance information does not belong to one element but to the combination of an operation and a resource.

This study defines the quality as an abstract level attribute, because the quality characteristics are very different from product to product and cannot be generalized. Although there are other elements and attributes that are not mentioned above like warehouse, part transfer vehicle, life-time of a resource, and so on, to keep the model compact and simple, relatively unimportant elements and attributes are not considered in this study.

\section{Modeling Methodology}

\subsection{Two-layered model: intuitive model and status-relationship model}

The extracted necessary information should be modeled from two kinds of perspectives: a business-side and a system-side (Fig.2). In general, field experts who have only business-side knowledge encounter difficulty in understanding systemoriented models because the information from the system-side perspective are too bizarre and complicated. Hence the system should be modeled in an intuitively understandable way from the business-side perspective. It helps field experts and system analysts to communicate with each other, which leads to improving the accuracy of the modeled information.

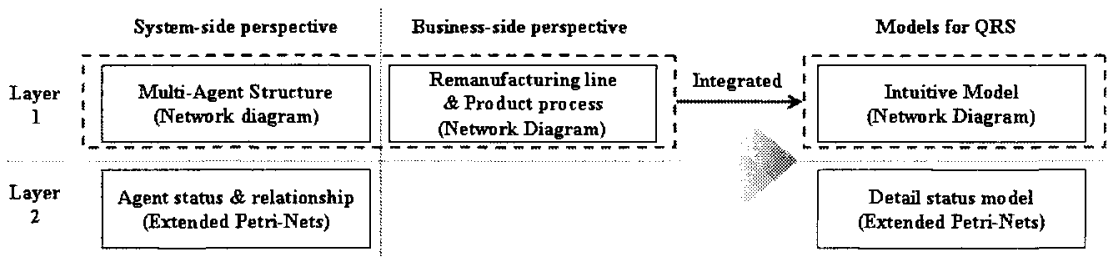

Fig. 2. Structure of Models for QRS.

From the business-side perspective, the above extracted necessary elements should be modeled as they are in the real world. Therefore, it is preferable to divide the business-side model into two kinds of models: a remanufacturing line model and product-process models, where work stations and buffers are modeled with their characteristics in a remanufacturing line model and operations of each product with disassembly relationships are modeled in the product-process models. For linking two models, information on resource allocation to operations and operation time and quality performance should be added. 
From the system-side perspective, we propose a two-layered structure, where the information representing the structure of a multi-agent framework for a QRS is modeled in the first layer, and the status of agents and their relationships are expressed in the second layer. This information can be surely expressed in an integrated model. However, the complexity of such an integrated model is very high, which leads to ambiguity in constructing the multi-agent structure.

Although modeling from two kinds of perspective is preferable, this study suggests integrating the model from the business-side perspective and the first layer model from the system-side perspective into one model (Fig.2), because they are similar in terms of the structure and contained information. In consequence, a twolayered model should is proposed for a QRS: an intuitive model and a statusrelationship model.

An intuitive model is composed of two parts like the model from the businessside perspective: remanufacturing line model and process model of products which flow on the line (product-process model). The relationship information between the two models should be added to integrate the two parts. Therefore the intuitive model can be defined as 4-tuples:

$$
\text { Intuitive model }=\left\{\boldsymbol{M}_{R}, \boldsymbol{M}_{P}, \lambda_{c s}, \boldsymbol{\Delta}_{q r}\right\}
$$

where $\boldsymbol{M}_{R}$ is a remanufacturing line model, $\boldsymbol{M}_{P}$ is a product-process model, $\lambda_{c s}$ is a resource allocation information, and $\boldsymbol{\Delta}_{q t}$ is a quality and time performance information depending on the combination of operations and resources.

A status-relationship model can be derived from XCPN, because it already has many features that we need as we explained above. The model can be defined as follows:

$$
\text { Status-relationship model }=\left\{P N^{T}{ }_{X C P N}, Q, P_{t}, P_{q}, C\right\}
$$

where $P N^{T}{ }_{X C P N}$ is a XCPN without time related feature, $Q$ is quality information of parts, $\boldsymbol{P}_{\boldsymbol{t}}$ and $\boldsymbol{P}_{\boldsymbol{q}}$ are time and quality performance information, and $\boldsymbol{C}$ is correlation information between operation time and quality. With existing features in XCPN, we can model the status of agents and their relationship.

The two models must support graphical notation. Modeling only with formal definition may cause much confusion for modelers as the system size increases. For this reason, we recommend modeling an intuitive model with a directed graph for a remanufacturing system in which overall time distribution of an operation and overall quality performance of a resource are expressed. For the status-relationship model, graphical notation of XCPN can be used with addition of symbols related with quality dependent part routing.

\subsection{Necessity of conversion method}

As we discussed in section 4.1, a two-layered model is preferable for applying a multi-agent approach to a QRS. An intuitive model has the basic structure information of a remanufacturing system. The status-relationship model has the supporting logic and characteristic information. It means that the latter should be constructed based on the information of the former, and the consistency between them has to be kept. 
To avoid inconsistency between them, it is necessary to develop a method that converts an intuitive model into a status-relationship model. The status-relationship model should contain additional information such as possible status and synchronization protocol of each element type, which do not exist in an intuitive model. Therefore the conversion method should provide such information. Hence the 'conversion' in this research means not only the change of expression but also the addition of pre-acquired information. For example, a resource agent should be able to have the status of waiting jobs, carrying a job on, examining an operation quality, and so on.

\subsection{Framework of QRS modeling and multi-agent system construction}

With the two-layered model and conversion method mentioned above, the real world information of a remanufacturing system can be modeled, and the information can be used for the construction of a multi-agent remanufacturing system in the virtual world. Fig.3 shows an overall framework for the modeling of a QRS and the constructing a multi-agent remanufacturing system based on the model.

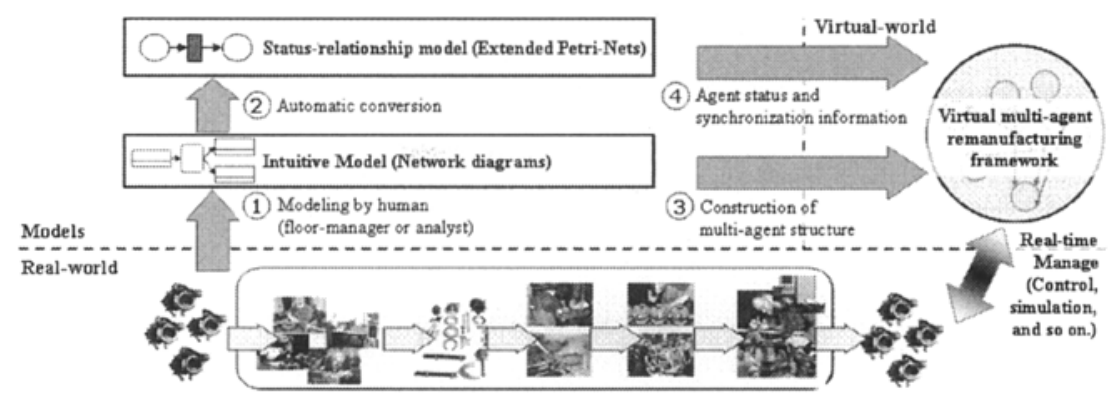

Fig. 3. Framework of modeling and constructing a multi-agent remanufacturing system.

The modeling and construction of a multi-agent remanufacturing system based on the model information can be done in 4 steps as follows:

Step 1. Create an intuitive model;

Step 2. Create a status-relationship model based on the conversion method;

Step 3. Construct a multi-agent structure in the virtual remanufacturing system;

Step 4. Load detailed status and synchronization information to each agent.

If the objective of the modeling is to control a remanufacturing system, each element in the constructed virtual remanufacturing system should be synchronized to the corresponding element in the remanufacturing system. But just for the simulation work, the synchronization is not needed. Instead of that, the virtual remanufacturing system must contain the module which can emulate real world situation and manage time. 


\section{Case Study}

For the better understanding of the proposed methodology, this section introduces a remanufacturing line example.
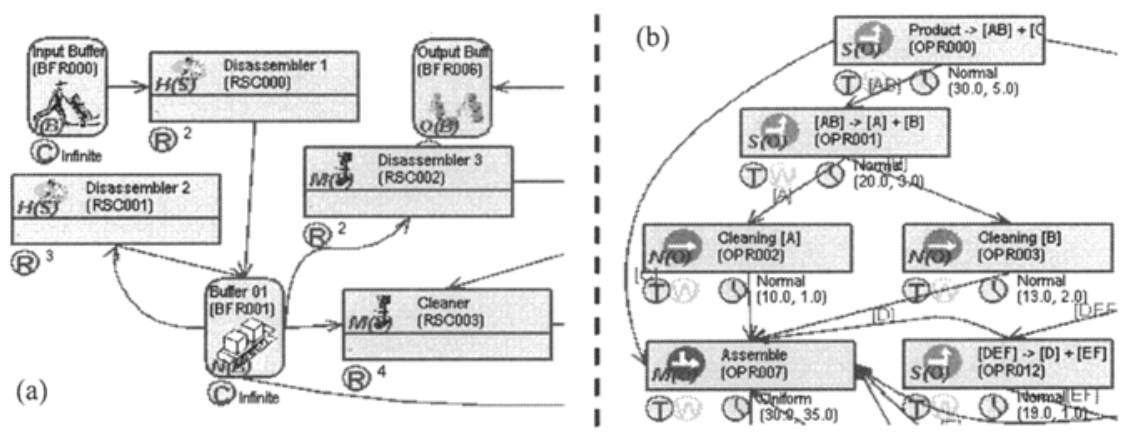

Fig. 4. Intuitive model; a part of (a) remanufacturing line and (b) operations of a product.

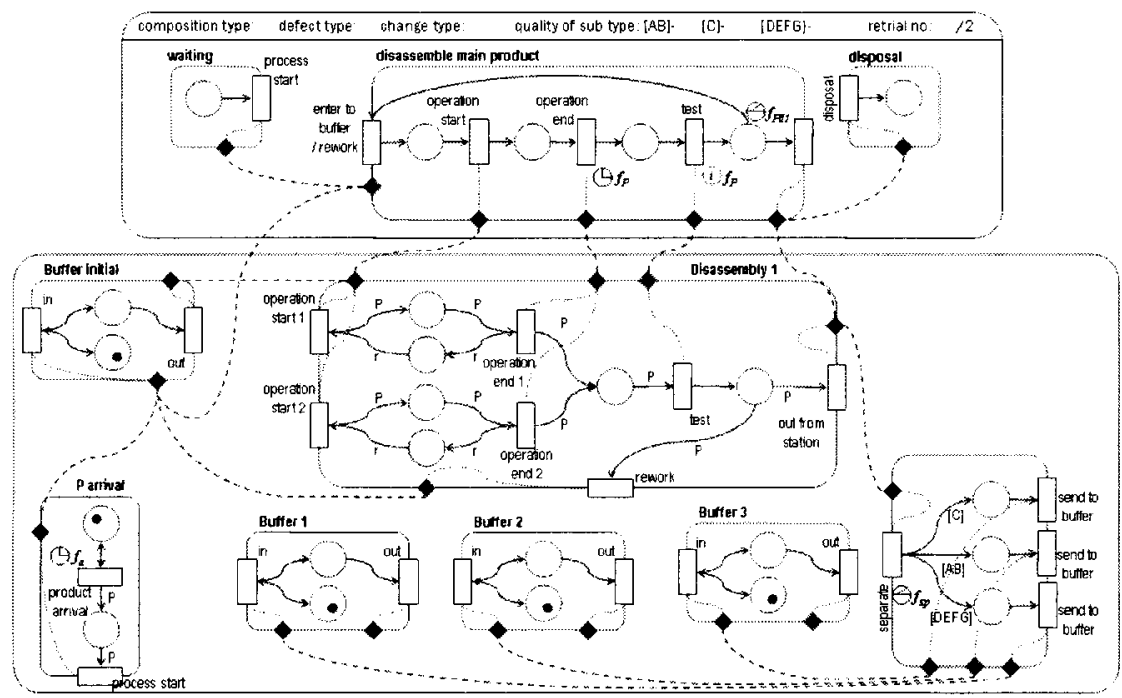

Fig. 5. A part of status-relationship model converted from the intuitive model in Fig.4.

Fig.4 is an example of an intuitive model. Fig.4(a) represents a part of the remanufacturing line and Fig.4(b) represents a part of the process of a product that flows on the line. It is designed by a directed graph which has various node types depending on element types such as resource, buffer, and operation in process. Fig. 5 shows a part of status-relationship model, which is converted by the pre-defined 
conversion method. In this figure, only partial buffers and work stations in the arrival point of a used product in the remanufacturing system are expressed. It is designed based on the XCPN notation. Some features which are not included in XCPN are added to represent dynamic quality and part routing. In the model, each workstation, buffer and operation is expressed as a module, and some modules are added for the agent life-cycle management.

\section{Conclusion}

In this research, we have proposed a methodology for modeling a QRS. In the methodology, a two-layered modeling approach has been suggested and the necessity of a conversion method has been explained. The proposed modeling framework enables us to represent a remanufacturing system well, considering its characteristics. Furthermore, the extracted necessary elements are helpful for enhancing the correctness of models. In spite of these contributions, there are still some limitations in this study: First, formal definition with graphical notation for intuitive model and detail specification for extended Petri-Nets should be done. Second, quality related features are preferable to be detailed and generalized based on analysis of many case studies.

\section{References}

1. V.D.R. Guide, M.E. Kraus, and R. Srivastava, Scheduling policies for remanufacturing, International Journal of Production Economics 48(2), 187-204 (1997)

2. W.M. Shen, Q. Hao, H.J. Yoon, and D.H. Norrie, Applications of Agent-based Systems in Intelligent Manufacturing: An Updated Review, Advanced Engineering Informatics 20(4), 415-431(2006).

3. L.S.H. Demello, and A.C. Sanderson, And/or graph representation of assembly plans, IEEE Transactions on Robotics and Automation 6(2), 188-199 (1990).

4. E. Zussman, and M.C. Zhou, A methodology for modeling and adaptive planning of disassembly processes, IEEE Transactions on Robotics and Automation 15(1), 190-194 (1999).

5. T.Y. ElMekkawy, and H.A. ElMaraghy, Real-time scheduling with deadlock avoidance in flexible manufacturing systems, International Journal of Advanced Manufacturing Technology 22(3-4), 259-270 (2003).

6. M Jeng, S.L. Xie, and M.Y. Peng, Process nets with resources for manufacturing modeling and their analysis, IEEE Transactions on Robotics and Automation 18(6), 875-889 (2002).

7. SIMAS II, CIMPACT (2000), available at http://www.cimpact.ch.

8. D. Sakara, Modeling and scheduling of remanufacturing systems, Ph.D. dissertation, STI-IPR-LICP, EPFL, Lausanne, Switzerland, 2006. 